\title{
Los límites entre el turismo y la migración residencial. Una tipología*
}

\author{
Raquel Huete \\ Alejandro Mantecón \\ Universidad de Alicante. Departamento de Sociología I \\ r.huete@ua.es \\ alejandro.mantecon@ua.es
}

Recibido: 09-03-2009

Aceptado: 17-09-2009

\section{Resumen}

En los procesos de movilidad internacional de jubilados desde el norte de Europa hasta las costas mediterráneas, se reconocen nuevos estilos de vida relacionados con fenómenos de naturaleza en ocasiones turística y a veces migratoria. Las sociedades que acogen a esta población requieren la definición de criterios para categorizar a estas personas como turistas o inmigrantes, pues la financiación y la gestión de sus recursos depende de ello. En este trabajo, se construye una tipología creada a partir de una encuesta realizada a 872 ciudadanos europeos en la provincia de Alicante. El empadronamiento y la forma de tenencia de la vivienda ocupada se revelan como las variables explicativas de la relación existente entre el turismo de larga estancia y la migración residencial.

Palabras clave: movilidad internacional, Mediterráneo, turismo residencial, jubilados, sociología.

Abstract. The Limits between Tourism and Residential Migration. A Typology

New lifestyles some related to tourism phenomena and others to migration trends, can be identified behind the international mobility process of retirees who move from northern European countries to Mediterranean areas. Host societies require the definition of criteria that will allow them to classify these people either as tourists or as immigrants, since the funding and management of their resources depend on it. In this article we propose a typology based on a survey of 872 European citizens in the province of Alicante. Both the fact of being registered in the local census and whether the dwelling is bought or rented by those European citizens turn out to be the explanatory variables of the relationship between long-stay tourism and residential migration.

Key words: international mobility, Mediterranean coast, residential tourism, retirees, sociology.

* Este trabajo es resultado del proyecto de investigación «La percepción social del turismo en la Comunidad Valenciana. Análisis sociológico y prospectiva (CSO2009-10293)», financiado por el Ministerio de Ciencia e Innovación. Los autores agradecen sus comentarios a los profesores Elena Jorge y Tomás Mazón. 


\author{
Sumario \\ 1. Introducción 4. Propuesta de una tipología \\ 2. Revisión de criterios y tipologías \\ 5. Conclusiones \\ 3. Metodología \\ Referencias bibliográficas
}

\title{
1. Introducción
}

Los procesos de movilidad internacional protagonizados por los jubilados que se trasladan desde los países europeos más fríos hacia las regiones mediterráneas han captado la atención de las ciencias sociales en los últimos años. Así, se han desarrollado líneas de investigación que abordan las motivaciones de los mayores para el traslado, las realidades geográficas que subyacen a sus pautas de movilidad, la formación de nuevas redes sociales y estructuras comunitarias en las sociedades de acogida o las valoraciones y las consecuencias que se derivan de los cambios de residencia (Barretto, 2008; Benson y O'Reilly, 2009; Gustafson, 2002a; Huber, 2005; Huete, 2005, 2009; King et al., 2000; O'Reilly, 2000; Rodríguez et al., 2005; Williams et al., 1997, 2000).

Simultáneamente, el concepto «turismo residencial» se ha utilizado desde finales de los años setenta para explicar los cambios sociales y ambientales causados por el incremento de dos formas de movilidad humana intensamente relacionadas en el contexto del mundo mediterráneo: el turismo y la migración. Los usuarios de las llamadas viviendas turístico-residenciales constituyen una amalgama social bajo la que se embuten casuísticas cada vez más diversas (Callejo et al., 2004; Huete et al., 2008; Jurdao, 1979; Leontidou y Marmaras, 2001; Mantecón, 2008a, 2008b; Mazón, 2006a, 2006b; Mazón y Aledo, 2005; Monreal, 2001; Salvà, 2002; O’Reilly, 2005, 2007a; Raya y Benítez, 2002; Rodríguez, 2004; Serrano, 2007; Vera, 2005). Al profundizar en esta realidad, el investigador se cuestiona la validez de una expresión que tipifica como turistas a personas con intención de permanecer indefinidamente en el «destino turístico» que, a menudo, poseen una vivienda en propiedad y están empadronadas. La alusión al turismo residencial como un modo de vida orientado al ocio en un entorno con condiciones ambientales mejores a las del lugar de origen resulta de provecho para las campañas publicitarias de los promotores inmobiliarios, pero se muestra insuficiente si lo que se pretende es estudiar una realidad social en la que se entremezclan formas de movilidad que dan lugar a multitud de tipos de turistas, residentes y migrantes.

El turismo se concibe como una evasión temporal de la normalidad cotidiana. Sin embargo, los protagonistas de esta investigación no buscan romper con el día a día asociado a la vida laboral. Se trata de retirados que pretenden redefinir su estilo de vida incorporando a la cotidianidad (uni-residencial, biresidencial o multi-residencial) un entorno distinto a aquel en el que han desarrollado su vida profesional. Por eso, no parece apropiado utilizar la etiqueta de «turismo residencial» para dar cuenta de los asentamientos de noreuropeos en 
el litoral mediterráneo. La explicación sociológica requiere de enfoques más flexibles, capaces de situar a estos procesos en un continuo que recoja una amplia gama de formas de movilidad cuya naturaleza, turística o migratoria, a menudo se torna borrosa (Barretto, 2008; Hall y Müller, 2004; King, 2002; O’Reilly, 2003; Urry, 2007; Williams y Hall, 2002).

El debate sobre la operatividad de la expresión «turismo residencial» no ha cesado desde que Francisco Jurdao publicara España en venta en 1979. Los investigadores en turismo y en economía del sector inmobiliario no alcanzan un consenso (Mazón y Aledo, 2005; Monreal, 2001; Salvà, 2002; Torres Bernier, 2003). El problema conceptual está íntimamente ligado al desarrollo de las migraciones intraeuropeas contemporáneas, cuyas dinámicas se han vuelto muy complejas (King, 2002; King et al., 1998; O’Reilly, 2003, 2007b; Williams et al., 1997, 2000). Se trata de decidir si las personas que residen durante periodos de tiempo más o menos prolongados en viviendas emplazadas en áreas turísticas, o en sus zonas de influencia, movidos por razones que tienen más que ver con la búsqueda de un lugar en el que disfrutar del ocio que con la realización de una actividad económica, han de ser consideradas inmigrantes, turistas residenciales o simplemente residentes.

La definición de la situación tiene aquí implicaciones cruciales para la planificación de extensos territorios. La gestión local se ve determinada por los recursos de los que se puede disponer y éstos se obtienen a partir de la información recogida en las estadísticas oficiales de residencialidad. El hecho de clasificar oficialmente a quienes se desplazan a un lugar como turistas o como inmigrantes afecta a la organización de los mercados de trabajo, la satisfacción de las necesidades educativas y sanitarias o la provisión de servicios básicos (suministro de electricidad, agua, recogida de basuras, etc.), entre otros aspectos de la gestión pública.

En las próximas páginas, se lleva a cabo una revisión de las clasificaciones presentadas durante los últimos años antes de exponer una nueva tipología que puede resultar válida a la hora de abordar la complejidad de tipos de movilidad residencial vinculada a motivaciones más relacionadas con el consumo de experiencias de ocio que con actividades productivas, y en la que los límites entre el turismo y la migración se diluyen. La investigación se realiza en un escenario paradigmático del Mediterráneo: la provincia de Alicante, también conocida en el sistema turístico como la Costa Blanca.

\section{Revisión de criterios y tipologías}

Algunos investigadores distinguen entre las migraciones por motivos laborales orientadas por la producción y las migraciones por motivos relacionados más estrechamente con el consumo, dentro de las cuales se incluirían los traslados a las segundas residencias, las migraciones estacionales, las migraciones por cambio en el estilo de vida (lifestyle migrations) y las migraciones de jubilados (Bell y Ward, 2000; Williams y Hall, 2000). De esta manera, el turismo se presenta orientado por el consumo de ocio en un continuo de movilidades 
voluntarias que comprende desde los viajes diarios de ida y vuelta al domicilio hasta algunos de los tipos que suelen clasificarse como migraciones. Esta conceptualización ayuda a integrar la investigación sobre el ocio con distintas formas de movilidad, por ejemplo: las vacaciones en las segundas residencias (Hall y Müller, 2004).

El grupo de investigación del Centro de Estudios de Inmigración y Minorías Étnicas de la Universidad Autónoma de Barcelona ha diferenciado cinco grupos de turistas o residentes según los motivos de su traslado (Moreno et al., 2004):

a) Turistas extranjeros jubilados (vienen a España a pasar sus vacaciones y, por lo tanto, permanecen un corto período de tiempo).

b) Inmigrados jubilados que han pasado de turistas a residentes (personas que veraneaban en estas localidades antes de la jubilación y que deciden instalarse definitivamente en el municipio buscando un lugar agradable donde pasar sus últimos años).

c) Jubilados comunitarios reagrupados por sus familias.

d) Personas que emigraron antes de la jubilación por otros motivos (trabajo, matrimonios mixtos, etc.) y que, más tarde, se jubilaron en España.

e) Jubilados que emigran para ingresar en una residencia, de forma temporal o permanente.

En relación con la mayor o menor voluntariedad del desplazamiento, Olivier Dehoorne (2002) señala que es la intencionalidad del traslado lo que denota la diferencia. El turismo correspondería a los traslados motivados por la búsqueda de placer, ocio, aventura o descubrimiento. Desde este punto de vista, las motivaciones son decisivas para distinguir las diferentes formas de movilidad. El grado de libertad es variable y da lugar a una gama de desplazamientos cada vez más amplia. Los determinantes socioeconómicos, políticos y familiares restringen la libertad en el caso de las migraciones, por lo cual su influencia, en comparación, es mucho menor en las decisiones acerca de los desplazamientos turísticos. Russell King (2002) también se refiere a la libertad como un posible criterio, pero pone en cuestión su utilidad para enfrentarse a los procesos residenciales que aquí se abordan cuando advierte sobre las altas cotas de libertad asociadas a las nuevas migraciones residenciales. De este modo, propone la distinción entre migración forzosa y migración voluntaria, y ejemplifica ésta última con el caso de los retirados que se asientan en las costas mediterráneas españolas.

Vicente Rodríguez (2001, 2004) y Toni Breuer (2005) son algunos de los investigadores que consideran que los comportamientos de los jubilados europeos, residentes estacionales, son susceptibles de ser caracterizados con rasgos turísticos particulares. Como cualquier turista que busca un espacio de vida distinto y complementario a su cotidianidad laboral durante un corto periodo de tiempo, los jubilados europeos, aunque no están atados a las obligaciones del mundo laboral, tratarían de aliviar los efectos de las estaciones climáticas más 
adversas a través del uso complementario de espacios que favorecen su salud. En concreto, el estudio de Breuer sobre el uso estacional de la residencia secundaria por parte de los alemanes retirados en las Islas Canarias le lleva a constatar el descubrimiento de «un nuevo estilo de vida»: «el turismo de residencia transnacional de personas en edad de jubilación» (2005: 372).

Charles Betty y Michael Cahill (1998) distinguen los siguientes tipos en su estudio sobre el acceso al sistema sanitario en la Costa del Sol:

a) Residentes inscritos (los que viven de forma más o menos permanente y están inscritos en el padrón).

b) Visitantes de larga duración (propietarios de vivienda, a menudo en urbanizaciones, que suelen utilizarla como segunda residencia en estancias de entre tres y seis meses).

c) Residentes no inscritos (viven casi todo el año y tienen vivienda en propiedad, pero, por diversas razones, no se inscriben en el padrón).

d) Arrendatarios (alquilan una vivienda y pasan en ella de tres a seis meses).

Javier Callejo, Jesús Gutiérrez y Antonio Viedma explican que «el significado y uso que se da a la vivienda, y no la ubicación o singularidad del inmueble, es lo que la define potencialmente como turística. En este sentido es oportuno comenzar señalando que objetivamente todas las viviendas particulares son susceptibles de considerarse como tal, si bien sólo sus inquilinos o usuarios pueden conferirle dicho papel» (2004: 145). Al respecto, Lars Aronsson (2004) identifica la residencia vacacional con un modo de vida que presupone tanto el deseo de movilidad como el de establecer vínculos de apego permanentes con el lugar en el que se localiza dicha residencia. El significado subjetivo que cada persona otorgue a esos deseos y a ese modo de vida decidirá qué significado tiene para ella esa residencia. David T. Duval (2004) precisa que ni la dimensión espacio-temporal ni el sistema de motivaciones que deciden la movilidad resultan suficientes para distinguir el turismo residencial de determinadas formas de migración, por lo que acaba por delegar la identificación del turista o del migrante en la propia definición de la identidad de cada individuo y de su sentido del hogar. De este modo, el apego al lugar de residencia, el compromiso con la sociedad de acogida y la vinculación con la sociedad de origen son también indicadores que pretenden calificar a las personas como inmigrantes o como turistas. Según Per Gustafson: «el apego a un lugar implica que el individuo está enraizado en un lugar y se encuentra identificado con su cultura y entorno social. Los conceptos de apego y movilidad no son opuestos; muchas personas con gran apego a un lugar presentan comportamientos muy móviles» (Gustafson, 2002b: 673). Coincide en esta idea Lars Aronsson: «desde el punto de vista de la relación entre movilidad y sentido de pertenencia a un lugar, relación cada vez más compleja en las sociedades modernas, los residentes temporales pueden ser estudiados como una categoría entre los turistas (móviles) y los residentes permanentes (adscritos al lugar)» (Aronsson, 2004: 75). Si se asumen las implicaciones de estas perspectivas, cualquier criterio 
Cuadro 1. Criterios que sirven para decidir la naturaleza turística o migratoria de las formas de movilidad habitualmente asociadas al turismo residencial

\begin{tabular}{|c|c|c|c|}
\hline Criterio & Turismo & Migraciones & Referencias básicas \\
\hline Motivaciones & $\begin{array}{l}\text { Libertad de elección. } \\
\text { Consumo. }\end{array}$ & $\begin{array}{l}\text { Razones económicas. } \\
\text { Producción. }\end{array}$ & $\begin{array}{l}\text { King, 2002; } \\
\text { Williams y Hall, 2000; } \\
\text { Dehoorne, 2002. }\end{array}$ \\
\hline \multirow[t]{3}{*}{ Temporalidad } & Eventualidad. & Permanencia. & Vera, 1990. \\
\hline & $\begin{array}{l}\text { Regreso a casa. } \\
\text { No hay registro } \\
\text { (empadronamiento). } \\
\text { Cambio de la residencia } \\
\text { eventual. }\end{array}$ & $\begin{array}{l}\text { Regreso al origen. } \\
\text { Trámites legales y } \\
\text { empadronamiento. } \\
\text { Cambio del entorno } \\
\text { cotidiano. }\end{array}$ & $\begin{array}{l}\text { Williams, King } \\
\text { y Warnes, } 1997 .\end{array}$ \\
\hline & $\begin{array}{l}\text { Menor tendencia } \\
\text { a la propiedad } \\
\text { de la vivienda. }\end{array}$ & $\begin{array}{l}\text { Mayor tendencia } \\
\text { a la propiedad } \\
\text { de la vivienda. }\end{array}$ & $\begin{array}{l}\text { King, Warnes y } \\
\text { Williams, } 2000\end{array}$ \\
\hline $\begin{array}{l}\text { Actividades y } \\
\text { estilos de vida }\end{array}$ & $\begin{array}{l}\text { Consumo de servicios } \\
\text { turísticos. }\end{array}$ & $\begin{array}{l}\text { Patrones de consumo } \\
\text { similares a los } \\
\text { autóctonos. }\end{array}$ & $\begin{array}{l}\text { Betty y Cahill, 1998; } \\
\text { Rodríguez, } 2004 .\end{array}$ \\
\hline \multirow[t]{4}{*}{$\begin{array}{l}\text { Formas de } \\
\text { relación social }\end{array}$} & $\begin{array}{l}\text { No hay integración } \\
\text { ni asimilación de } \\
\text { costumbres. }\end{array}$ & $\begin{array}{l}\text { Se espera la integración } \\
\text { y una cierta asimilación } \\
\text { de las costumbres. }\end{array}$ & Jaakson, 1986. \\
\hline & $\begin{array}{l}\text { Autopercepción } \\
\text { como turistas. }\end{array}$ & $\begin{array}{l}\text { Autopercepción como } \\
\text { no turistas. }\end{array}$ & $\begin{array}{l}\text { Gustafson, 2002a; } \\
\text { Duval, } 2004 .\end{array}$ \\
\hline & $\begin{array}{l}\text { No hay compromiso } \\
\text { ni sentido de } \\
\text { pertenencia. }\end{array}$ & $\begin{array}{l}\text { Se espera cierto } \\
\text { compromiso y sentido } \\
\text { de pertenencia. }\end{array}$ & $\begin{array}{l}\text { O’Reilly, 1995; } \\
\text { Gustafson, 2002a; } \\
\text { Müller, 2002; } \\
\text { Aronsson, 2004; } \\
\text { Duval, 2004. }\end{array}$ \\
\hline & $\begin{array}{l}\text { Mantenimiento del } \\
\text { «espacio de vida» } \\
\text { habitual. }\end{array}$ & $\begin{array}{l}\text { Ruptura con el «espacio } \\
\text { de vida» habitual. }\end{array}$ & Thumerelle, 1986. \\
\hline
\end{tabular}

Fuente: elaboración propia.

externo a la subjetividad del residente puede derivar en situaciones en las que un alto porcentaje de personas no se identifique con la etiqueta asignada a su vivienda y que, en consecuencia, se las tipifique como turistas o migrantes cuando no se reconozcan a sí mismas en uno de estos grupos o en ninguno. En todo caso, las autoridades responsables precisan de la utilización de tipologías operativas, si bien intentando que éstas muestren una asimetría lo más atenuada posible respecto a la percepción subjetiva que tienen de su situación los protagonistas de las nuevas formas residenciales.

En el cuadro 1, se sintetizan algunas de las aproximaciones más habituales con las que se ha tratado de marcar la diferencia entre los procesos de movilidad comentados.

Los estudios realizados en las regiones turísticas mediterráneas revelan varios grupos sociales que, en algún momento, han sido etiquetados como turistas 
residenciales. El primero está integrado por los veraneantes: turistas que, en periodo vacacional, se trasladan a un apartamento (prestado, alquilado o del que son propietarios) en el que disfrutan de un tiempo de ocio. Sobre todo se hace referencia a las familias españolas que cada verano hacen que la población de los enclaves turísticos se incremente de forma espectacular (Callejo et al., 2004). No cabe duda de que la naturaleza de la estancia de estos veraneantes es turística, del mismo modo que lo es la de quienes optan por alojarse en un hotel, un hostal o un camping. El segundo grupo es mucho más heterogéneo. Tras completar una investigación etnográfica sobre las formas de vida de los jubilados británicos asentados en la Costa del Sol, Karen O’Reilly (2007b) distingue los tipos siguientes: por un lado, un grupo principal de migrantes retirados, que se subdivide en permanentes, temporales y estacionales, y, por otro lado, dos grupos muy reducidos de migrantes empresariales (propietarios de pequeños negocios que ofrecen diferentes servicios a los residentes noreuropeos) y de migrantes económicamente activos (casos excepcionales de individuos que realizan su trabajo desde la distancia o yendo y viniendo desde sus países hasta España). El grupo principal de los migrantes retirados acoge a una amplia variedad de casos. De tal manera que si, en el subgrupo de los "permanentes», se pueden observar rasgos más parecidos a los que se reconocen en los procesos migratorios que en los turísticos, en los subgrupos de "temporales» y "estacionales» ocurre lo contrario. La misma autora había publicado dos años antes (O’Reilly, 2005) otra clasificación basada en la combinación de la orientación que la persona tenga hacia uno u otro país y el tiempo que pasa en ellos:

a) Residentes permanentes: son los inmigrantes, jubilados y trabajadores.

b) Residentes de retorno: residentes en España por lo que se refiere a su estatus legal, pero que regresan a Gran Bretaña entre dos y cinco meses al año (cada verano).

c) Visitantes estacionales: viven en Gran Bretaña pero regresan a España cada invierno. La diferencia cualitativa entre este grupo y el de los residentes es básicamente la orientación, que tiende más hacia su país que al de acogida.

d) Visitantes ocasionales: poseen normalmente una segunda residencia que visitan por tener propiedades, familia, amigos o negocios en España.

Russell King, Anthony Warnes y Allan Williams (2000) confirman la importancia de la propiedad de la vivienda y la duración de la estancia en el país de destino como los elementos esenciales para diferenciar entre turistas e inmigrantes jubilados, y proponen otra clasificación:

a) Turistas internacionales de larga estancia que normalmente pasan los inviernos en el sur, donde alquilan una casa.

b) Propietarios de segunda residencia que pasan periodos relativamente cortos en los destinos mediterráneos.

c) Inmigrantes estacionales, quienes pasan una estancia relativamente larga que puede o no ir acompañada del empadronamiento. 
d) Residentes permanentes que viven todo el año en el Mediterráneo y realizan visitas o vacaciones a otros lugares.

En esta tipología, los autores reconocen el interés de considerar el registro como una variable fundamental, al tiempo que prestan una atención específica a la duración de la estancia, pero, al igual que ocurre con el resto de las clasificaciones revisadas, no realizan las pruebas de estadísticas necesarias para garantizar la consistencia empírica de los tipos propuestos.

Precisamente, la carencia de una tipología cuyos criterios puedan operativizarse en variables de respuesta objetiva y generalizable en distintos contextos sirve de impulso a este trabajo. Por consiguiente, el objetivo aquí es presentar una propuesta tipológica que explique las diferentes situaciones de aquellos ciudadanos europeos jubilados que se alojan en viviendas situadas en regiones turísticas por periodos de tiempo más o menos largos y que la sociedad anfitriona denomina mayoritariamente «turistas residenciales» (Huete et al., 2008).

\section{Metodología}

La investigación de los movimientos turísticos y las migraciones residenciales mediante encuesta presenta importantes dificultades metodológicas debido, fundamentalmente, a la ausencia de un marco muestral. Las encuestas anteriores sobre esta población se realizaron por medio de muestreos de conveniencia (Breuer, 2005; Casado, 1999; Huber, 2005; Williams y Hall, 2002) o mediante muestreo por cuotas no probabilístico (Rodríguez, FernándezMayoralas y Rojo, 1998). En el presente estudio, el intento por realizar una investigación con vocación de representatividad supone un avance significativo en el conocimiento empírico de los procesos de movilidad internacional por motivos residenciales. El diseño muestral y la aplicación cara a cara en el hogar de los entrevistados sustentan la validez de la encuesta. Para la selección de las unidades muestrales, los municipios de la provincia de Alicante se agruparon en tres conglomerados, según su disposición geográfica respecto de la costa (litoral, prelitoral e interior), y de cada conglomerado se seleccionaron los cuatro municipios con mayor número de viviendas construidas durante el período 2002-2003. De esa manera, se intentaba obtener una muestra de aquellos municipios en los que se había manifestado la actividad de construcción residencial más intensa en los años anteriores a la realización de la encuesta. El universo poblacional estaba compuesto por ciudadanos nacionales de alguno de los 25 países pertenecientes a la Unión Europea en 2005, excepto España, en situación de inactividad laboral (jubilados, pensionistas y amas de casa), alojados en vivienda particular, y fue objeto de un muestreo aleatorio simple con afijación proporcional en función de la citada disposición geográfica y los datos del Padrón municipal de habitantes de 2003. Se realizaron 872 entrevistas: 406 en municipios costeros (Denia, Benidorm, Santa Pola y Torrevieja), 409 en el prelitoral —localidades situadas a menos de 20 kilómetros de la costa- 
(Pedreguer, La Nucía, San Fulgencio y Rojales) y 57 en poblaciones localizadas a mayor distancia (Hondón de los Frailes, Aspe, Castalla y Biar). El error muestral es de $+/-5 \%$ para un nivel de confianza del $95,5 \%(p=q=50)$. El trabajo de campo se llevó a cabo en el primer trimestre de 2005. La selección última del hogar del entrevistado se realizó mediante rutas aleatorias a partir de cuadrículas que se establecieron según los mapas municipales obtenidos en cada uno de los ayuntamientos. La complejidad de los movimientos de personas, tal y como ha quedado reflejada en las páginas anteriores, obligó a tomar decisiones metodológicas que permitieran dejar lo más abiertas posible las diferentes estrategias residenciales. Por ello, no se establecieron limitaciones en cuanto a la estancia mínima en el lugar de realización de la entrevista ni en cuanto a la edad de los entrevistados y se asumió que existe una variación estacional de los efectivos, por lo que se decidió realizar el trabajo de campo en el primer trimestre del año, momento de menor afluencia turística. El cuestionario se aplicó en los idiomas castellano, francés, inglés y alemán. Dicho cuestionario recogía tres grandes bloques de preguntas: perfil sociodemográfico, motivos para el traslado y definición del estilo de vida en tanto que factor de motivación para la selección de la provincia de Alicante como destino residencial. Los límites que impone la extensión de un artículo de investigación obligan a que, a continuación, se enuncien únicamente las variables analizadas en este trabajo:

a) Perfil sociodemográfico: nacionalidad, edad, estudios cursados, ocupación profesional anterior, meses al año que reside en el municipio, veces al año que se desplaza a su país de origen, empadronamiento, propiedad de vivienda en el país de origen, tenencia de la vivienda (propiedad, multipropiedad, alquilada, familiares o amigos).

b) Motivos para el traslado: motivo principal para el traslado a la Costa Blanca (respuesta abierta recodificada en: clima, calidad de vida, conocimiento previo, otros) y calidad de vida percibida en comparación con el lugar de origen.

c) Apego al lugar: regreso al país en caso de enfermedad y grado de acuerdo con las siguientes afirmaciones (escala tipo Likert de 1 a 5 ): «La Administración española es muy complicada»; «No me hace falta saber español para desenvolverme»; «En los últimos años, hay más delincuencia»; "No tengo información suficiente para desenvolverme por mí mismo/a»; "Me gustaría aprender español»; «Es muy difícil conocer españoles», $\mathrm{y}$ «El turismo es beneficioso para el paisaje».

\section{Propuesta de una tipología}

La nacionalidad con mayor número de efectivos es la británica, representada por un $58,7 \%$ de los entrevistados; la siguiente es la alemana, con un porcentaje del $14,3 \%$. Un tercer grupo queda formado por el conglomerado del resto de nacionalidades, que alcanza el $26,9 \%$ de la población. La media de edad es 
de 62,6 años; 64,9 para los varones y 59,8 las mujeres. El nivel de estudios es medio y alto: el $44,5 \%$ tiene estudios universitarios, mientras que el $42,4 \%$ cursó estudios secundarios. La ocupación anterior declarada es acorde con los niveles de estudios, destacan un 12,7\% de directivos de empresas y un 14,3\% de técnicos y científicos.

En referencia a las pautas de residencialidad, el 59,3\% de la población vive prácticamente todo el año (diez meses o más) en la provincia de Alicante, mientras que apenas un 10,9\% realiza estancias de menos de tres meses; el 29,8\% restante permanece en la región entre cuatro y nueve meses. La frecuencia de las visitas a los países de los que proceden puede ser un indicador de los vínculos que los retirados mantienen con sus hogares y está estrechamente relacionada con el grado de asentamiento en la comunidad receptora. Por ello, al indagar sobre cuántas veces al año vuelven, se comprueba que el 68,3\% regresa pocas veces (en una o dos ocasiones cada año) y son pocos quienes presentan una alta movilidad (únicamente el 7,2\% del total realiza más de cuatro viajes anuales). No se halló una relación significativa entre el número de veces que una persona se desplaza a su país de origen y la estancia media en él. Sin embargo, sí se constató que aquellos que permanecen más meses son los que menos viajan. $\mathrm{Al}$ respecto, podría deducirse que los viajes al país de origen son para pasar temporadas largas, más que para visitas de corta duración.

Casi el 68\% de los entrevistados declara estar inscrito oficialmente en los registros de los ayuntamientos. Este porcentaje en el empadronamiento coincide con las aproximaciones realizas por otros investigadores (Casado et al., 2004; La Parra y Mateo, 2008) y no llegan a cuatro de cada diez los que mantienen casa en sus países. Es éste un asunto importante a la hora de avanzar en el perfil de la población, pues, sin casa en el país de origen, difícilmente se puede hablar de semirresidencialidad o bilocalidad, y aún menos de turismo. Un argumento a tener en cuenta a la hora de considerar este resultado es que, según otros estudios sobre migraciones internacionales de retirados (Warnes et al., 2004), muchos jubilados financian el traslado residencial con la renta obtenida de la venta de su casa ubicada normalmente en zonas revalorizadas de las metrópolis europeas. Con este capital pueden adquirir una vivienda en la zona de destino y, además, guardar unos ahorros que les facilitan la mejora de su calidad de vida. Ante la evidencia de que son muchos los que no tienen una vivienda en origen, una pregunta relevante es: ¿cuál es el régimen de tenencia de la casa de que disfrutan en la Costa Blanca? Los resultados son abrumadores: el $85,5 \%$ son propietarios, el $10,9 \%$ residen en viviendas alquiladas, el 3,4\% se hospeda en casas de familiares o amigos y el 2,2\% reside en una vivienda con tenencia de multipropiedad.

La información recogida demuestra que no hay una relación significativa entre haber visitado previamente la Costa Blanca como turista y poseer una vivienda en propiedad. Puede resultar insólito que un 26,9\% (190) de las personas que tienen vivienda propia declare no haber visitado la zona como turista. Estos datos cuestionan las tesis habituales que afirman que la compra de la vivienda en el destino turístico es la consecuencia de un proceso previo de fide- 
lización del turista al destino. Así pues, no se pueden hacer generalizaciones en las que se indique que el conjunto de tipos de movilidad residencial que habitualmente se asocian a la etiqueta "turista residencial» son turistas «fidelizados» con vivienda en propiedad, o que son el resultado de un turista vacacional satisfecho que encontró su vivienda secundaria durante una o varias estancias vacacionales.

Esto nos lleva a indagar sobre la relación entre la forma de tenencia de la vivienda en la Costa Blanca y el hecho de mantener o no una vivienda en el país de origen. Existe una relación de dependencia significativa $\left(\chi^{2}=46,220\right.$; $p<0,0001)$ entre ambas variables que demuestra que los propietarios de vivienda tienden a no mantener otra casa en mayor medida que quienes no lo son. El 66,6\% de los propietarios no mantiene una casa en su país frente a quienes se alojan en régimen de alquiler $(42,1 \%)$, multipropiedad $(35,5 \%)$ o en casa de familiares o amigos $(34,5 \%)$.

El análisis de los datos hasta aquí expuestos conduce a plantear la hipótesis de que la tipología resultante del cruce entre empadronamiento y tenencia de la vivienda es la mejor representación de cómo el binomio formado por turismo y migración se distribuye en la provincia de Alicante. Se llamará a la nueva variable "tipo de movilidad residencial por motivos de ocio», cuyas categorías son las siguientes:

- Tipo 1. Residentes permanentes: aquellos extranjeros que poseen vivienda en propiedad y que están registrados en el padrón municipal de habitantes.

- Tipo 2. Residentes temporales: aquellos extranjeros que se alojan en una vivienda que no es de su propiedad, la tienen en régimen de alquiler o multipropiedad o es de amigos o familiares, y que están registrados en el padrón municipal de habitantes.

- Tipo 3. Propietarios de vivienda secundaria: aquellos extranjeros que poseen una vivienda en propiedad pero que no están empadronados.

- Tipo 4. Turistas estacionales: aquellos extranjeros que ni poseen vivienda en propiedad ni están registrados en el padrón municipal de habitantes.

Se ha decidido, por motivos analíticos, agrupar las categorías «alquiler», «multipropiedad» $\mathrm{y}$ «familiares y amigos» en una sola categoría que equivaldría a la «no propiedad». Como se refleja en la tabla 1, el tipo que se ha denominado «residente permanente» (propietario empadronado) es el más numeroso, pues engloba al $61,7 \%$ de la población objeto de estudio. En el otro extremo, el «turista estacional» (no propietario ni empadronado) apenas aglutina a una décima parte de los entrevistados (10,3\%). A los otros dos tipos se les ha llamado «residente temporal» (no propietario registrado) y "propietario de segunda residencia» (propietario no empadronado).

Al comprobar cómo estos tipos se distribuyen según la nacionalidad (tabla 2), se observa que la propiedad es más frecuente entre los británicos, mientras que son los ciudadanos del resto de países de la UE quienes más tienden al alquiler y aún más al alquiler sin empadronamiento, esto es, a seguir la 
Tabla 1. Tipología de movilidad residencial por motivos de ocio

\begin{tabular}{lccc}
\hline & \multicolumn{3}{c}{ Tenencia de la vivienda } \\
\cline { 2 - 4 } Empadronamiento & Propiedad & Multipropiedad, alquiler o amigos & Total \\
\hline Sí & $61,7 \%$ & $6,2 \%$ & $67,9 \%$ \\
& $(531)$ & $(53)$ & $(584)$ \\
& Residentes & Residentes & \\
\hline permanentes & temporales & $32,1 \%$ \\
& $21,7 \%$ & $10,3 \%$ & $(276)$ \\
& $(187)$ & $(89)$ & \\
\hline Total & Propietarios de & Turistas estacionales & $\mathbf{1 0 0 , 0}$ \\
& vivienda secundaria & & $(\mathbf{8 6 0 )}$ \\
\hline$\chi^{2}=71,768 ; P<0,000$ & $\mathbf{1 6 , 5 \%}$ &
\end{tabular}

$\chi^{2}=71,768 ; p<0,000$.

$N=860$.

Fuente: elaboración propia a partir de la encuesta.

Tabla 2. Tipología de movilidad residencial por motivos de ocio y nacionalidad

\begin{tabular}{|c|c|c|c|c|c|}
\hline \multirow[b]{2}{*}{ Empadronamiento } & \multirow{2}{*}{$\begin{array}{l}\text { Tenencia } \\
\text { de vivienda }\end{array}$} & \multicolumn{4}{|c|}{ Nacionalidad } \\
\hline & & Alemania & Resto UE & Reino Unido & Total \\
\hline \multirow[t]{2}{*}{ Sí } & $\begin{array}{l}\text { Propiedad } \\
\text { Residentes } \\
\text { permanentes }\end{array}$ & $\begin{array}{c}14,9 \% \\
(79)\end{array}$ & $\begin{array}{c}22,0 \% \\
(117)\end{array}$ & $\begin{array}{c}63,1 \% \\
(335)\end{array}$ & $\begin{array}{l}100 \% \\
(531)\end{array}$ \\
\hline & $\begin{array}{l}\text { Multipropiedad, } \\
\text { alquiler o amigos } \\
\text { Residentes } \\
\text { temporales }\end{array}$ & $\begin{array}{l}17,0 \% \\
(9)\end{array}$ & $\begin{array}{c}45,3 \% \\
(24)\end{array}$ & $\begin{array}{c}37,7 \% \\
(20)\end{array}$ & $\begin{array}{c}100 \% \\
(53)\end{array}$ \\
\hline \multirow[t]{2}{*}{ No } & $\begin{array}{l}\text { Propiedad } \\
\text { Propietarios } \\
\text { de vivienda } \\
\text { secundaria }\end{array}$ & $\begin{array}{c}13,4 \% \\
(25)\end{array}$ & $\begin{array}{c}25,1 \% \\
(47)\end{array}$ & $\begin{array}{c}61,5 \% \\
(115)\end{array}$ & $\begin{array}{l}100 \% \\
(187)\end{array}$ \\
\hline & $\begin{array}{l}\text { Multipropiedad, } \\
\text { alquiler o amigos } \\
\text { Turistas estacionale }\end{array}$ & $\begin{array}{c}12,4 \% \\
(11)\end{array}$ & $\begin{array}{c}49,4 \% \\
(44)\end{array}$ & $\begin{array}{c}38,2 \% \\
(34)\end{array}$ & $\begin{array}{c}100 \% \\
(89)\end{array}$ \\
\hline
\end{tabular}

Si empadronado $\chi^{2}=16,026 ; \mathrm{p}<0,0001$.

No empadronado: $\chi^{2}=16,912 ; \mathrm{p}<0,0001$.

$N=860$.

Fuente: elaboración propia a partir de la encuesta.

pauta más temporal de las cuatro categorías. Los alemanes se distribuyen más homogéneamente entre las cuatro situaciones, aunque su presencia es mayor entre los residentes temporales.

Las personas que mantienen una vivienda en sus países a pesar de ser propietarios y estar empadronados en España, no llegan a la cuarta parte de los 
Tabla 3. Tipología de movilidad residencial por motivos de ocio y mantenimiento de vivienda en origen

\begin{tabular}{|c|c|c|c|c|}
\hline \multirow[b]{2}{*}{ Empadronamiento } & \multirow{2}{*}{$\begin{array}{l}\text { Tenencia } \\
\text { de vivienda }\end{array}$} & \multicolumn{3}{|c|}{ ¿Mantiene una casa en su país de origen? } \\
\hline & & Sí & No & Total \\
\hline \multirow[t]{2}{*}{ Sí } & $\begin{array}{l}\text { Propiedad } \\
\text { Residentes } \\
\text { permanentes }\end{array}$ & $\begin{array}{c}23,8 \% \\
(124)\end{array}$ & $\begin{array}{c}76,2 \% \\
(398)\end{array}$ & $\begin{array}{l}100 \% \\
(522)\end{array}$ \\
\hline & $\begin{array}{l}\text { Multipropiedad, } \\
\text { alquiler o amigos } \\
\text { Residentes } \\
\text { temporales }\end{array}$ & $\begin{array}{c}43,4 \% \\
(23)\end{array}$ & $\begin{array}{c}56,6 \% \\
(30)\end{array}$ & $\begin{array}{c}100 \% \\
(53)\end{array}$ \\
\hline \multirow[t]{2}{*}{ No } & $\begin{array}{l}\text { Propiedad } \\
\text { Propietarios } \\
\text { de vivienda } \\
\text { secundaria }\end{array}$ & $\begin{array}{l}60,9 \% \\
(112)\end{array}$ & $\begin{array}{c}39,1 \% \\
(72)\end{array}$ & $\begin{array}{l}100 \% \\
(184)\end{array}$ \\
\hline & $\begin{array}{l}\text { Multipropiedad, } \\
\text { alquiler o amigos } \\
\text { Turistas estacionales }\end{array}$ & $\begin{array}{c}76,1 \% \\
(67)\end{array}$ & $\begin{array}{c}23,9 \% \\
(21)\end{array}$ & $\begin{array}{c}100 \% \\
(88)\end{array}$ \\
\hline
\end{tabular}

Si empadronado $\chi^{2}=9,754 ; \mathrm{p}<0,0002$.

No empadronado: $\chi^{2}=6,166 ; \mathrm{p}<0,013$.

$N=847$.

Fuente: elaboración propia a partir de la encuesta.

entrevistados. Un porcentaje similar representan quienes no tienen casa en sus países pero tampoco en la Costa Blanca ni están empadronados (tabla 3).

La razón principal de elegir la Costa Blanca como destino residencial se planteó en forma de pregunta abierta, cuya respuesta fue transcrita literalmente por el entrevistador y posteriormente recodificada. De este modo, las diferentes expresiones para referirse al buen tiempo de la zona o a los días soleados que se disfrutan en la Costa Blanca fueron codificados bajo la categoría "clima». En la categoría "calidad de vida" se incluyeron expresiones variadas que tienen que ver con la salud, el estilo de vida, el ambiente, la tranquilidad y la gente española. Conocer la zona por haberla visitado como turista vacacional previamente al traslado se incorporó como una tercera categoría, y en la categoría «otros» se incluyeron diferentes respuestas, como las que aludían a precios baratos de la vivienda o el nivel de vida, conocer gente en la región o que hubiera facilidades para practicar el golf. El clima es el motivo para el traslado que declaran en mayor medida los propietarios de segunda residencia y los turistas estacionales. Sin embargo, la calidad de vida importa más a los residentes, tal y como se puede comprobar en la tabla 4. El conocimiento previo del lugar, bien por haberlo visitado como turista o bien porque ya vivían conocidos o familiares en él, es más relevante para los residentes temporales que para los otros tipos encontrados.

Por otra parte, el 71,8\% de los entrevistados agrupado en la categoría «residentes permanentes» está de acuerdo con la afirmación «Aquí disfruto de una 
Tabla 4. Tipología de movilidad residencial por motivos de ocio y motivo principal para el traslado

\begin{tabular}{|c|c|c|c|c|c|c|}
\hline \multirow[b]{2}{*}{ Empadronamiento } & \multirow[b]{2}{*}{$\begin{array}{l}\text { Tenencia } \\
\text { de vivienda }\end{array}$} & \multicolumn{5}{|c|}{ Motivo principal para el traslado a la Costa Blanca } \\
\hline & & Clima & $\begin{array}{l}\text { Calidad } \\
\text { de vida }\end{array}$ & $\begin{array}{c}\text { Conocimiento } \\
\text { previo }\end{array}$ & Otros & Total \\
\hline \multirow[t]{2}{*}{ Sí } & $\begin{array}{l}\text { Propiedad } \\
\text { Residentes } \\
\text { permanentes }\end{array}$ & $\begin{array}{l}50,9 \% \\
(266)\end{array}$ & $\begin{array}{l}38,9 \% \\
(204)\end{array}$ & $\begin{array}{c}3,4 \% \\
(18)\end{array}$ & $\begin{array}{l}6,7 \% \\
(35)\end{array}$ & $\begin{array}{l}100 \% \\
(522)\end{array}$ \\
\hline & $\begin{array}{l}\text { Multipropiedad, } \\
\text { alquiler o amigos } \\
\text { Residentes } \\
\text { temporales }\end{array}$ & $\begin{array}{l}39,6 \% \\
(21)\end{array}$ & $\begin{array}{c}32,1 \% \\
(17)\end{array}$ & $\begin{array}{l}17,0 \% \\
(9)\end{array}$ & $\begin{array}{c}11,3 \% \\
(6)\end{array}$ & $\begin{array}{c}100 \% \\
(53)\end{array}$ \\
\hline \multirow[t]{2}{*}{ No } & $\begin{array}{l}\text { Propiedad } \\
\text { Propietarios } \\
\text { de vivienda } \\
\text { secundaria }\end{array}$ & $\begin{array}{l}65,3 \% \\
(120)\end{array}$ & $\begin{array}{c}25,0 \% \\
(46)\end{array}$ & $\begin{array}{l}5,7 \% \\
(10)\end{array}$ & $\begin{array}{c}4,0 \% \\
(7)\end{array}$ & $\begin{array}{l}100 \% \\
(184)\end{array}$ \\
\hline & $\begin{array}{l}\text { Multipropiedad, } \\
\text { alquiler o amigos } \\
\text { Turistas estacionales }\end{array}$ & $\begin{array}{l}54,2 \% \\
(48)\end{array}$ & $\begin{array}{c}32,7 \% \\
(29)\end{array}$ & $\begin{array}{c}6,6 \% \\
(6)\end{array}$ & $\begin{array}{c}6,6 \% \\
(6)\end{array}$ & $\begin{array}{c}100 \% \\
(88)\end{array}$ \\
\hline
\end{tabular}

$\chi^{2}=72,071 ; \mathrm{p}<0,000$.

$N=847$.

Fuente: elaboración propia a partir de la encuesta.

mayor calidad de vida que en mi país de origen», frente al 62,9\% de los «propietarios de segunda residencia», el 55,8\% de los «residentes temporales» y el $51,2 \%$ de los «turistas residenciales» $\left(\chi^{2}=24,113, p<0,0001\right)$. De tal manera, podría afirmarse que las personas entrevistadas que se hallan más vinculadas a la Costa Blanca, considerando las variables básicas "empadronamiento" $\mathrm{y}$ «tenencia de la vivienda», son las que, igualmente, reconocen disfrutar de una mayor calidad de vida en la región.

En las siguientes líneas, se pretende comprobar la hipótesis de que el apego al lugar es una variable explicativa de la diferencia entre el turista y el inmigrante (Aronsson, 2004; Duval, 2004; Gustafson, 2002a; Jaakson, 1986; O'Reilly, 1995; Müller, 2002) y, también, si se encuentran diferencias consistentes con la tipología basada en la tenencia de la vivienda y el empadronamiento. Esta verificación se realiza mediante el análisis de los resultados sobre el grado de acuerdo que los entrevistados declaran a una serie de afirmaciones relacionadas con la integración en la sociedad de acogida y la vida cotidiana. Es lógico esperar una mayor vinculación de los residentes y una menor de los turistas con la región mediterránea en la que pasan temporadas más o menos largas, pero aquí se trata de contrastar empíricamente dicha afirmación. El aprendizaje del idioma es el primer paso para establecer un vínculo con la sociedad de acogida. El 27,8\% de los entrevistados declara que le gustaría aprender español, y los «residentes permanentes» $(29,5 \%)$ son quienes más de acuerdo están $\left(\chi^{2}=12,642 ; p=0,049\right)$. Asimismo, el $25,8 \%$ del total dice que no 
Cuadro 2. Características básicas de las estrategias de movilidad residencial por motivos de ocio identificadas

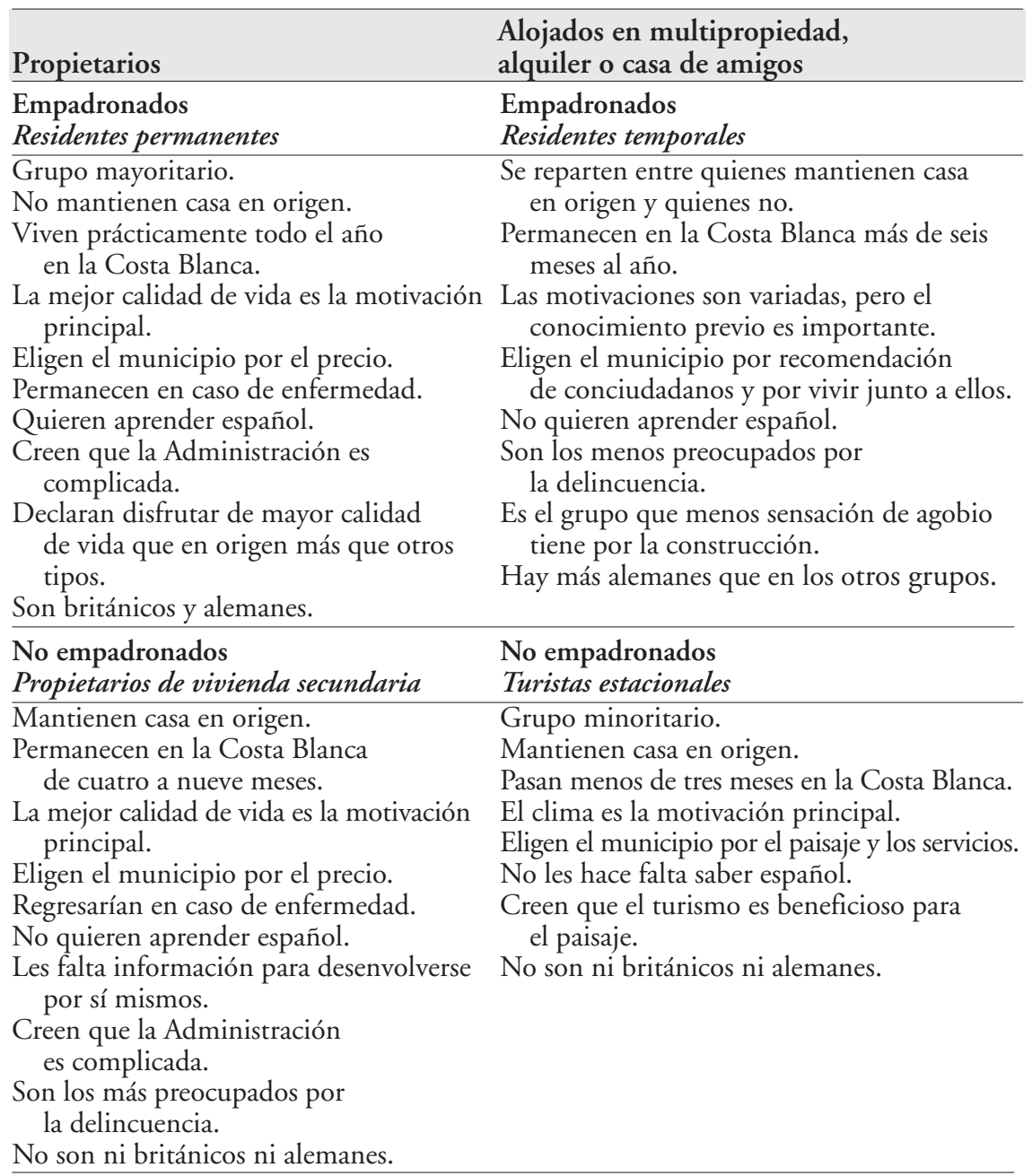

Fuente: elaboración propia.

necesita saber español para desenvolverse en el día a día, pero los «residentes permanentes» se ajustan menos a esta afirmación $(23,8 \%)$, al tiempo que es el tipo "turista estacional» el que en mayor medida declara que no le hace falta conocer el idioma $(37,6 \%)\left(\chi^{2}=19,468 ; p=0,003\right)$. Una mayor permanencia en Alicante implica una mayor conciencia de los impactos negativos del turismo sobre el medio ambiente, puesto que el 45\% de los «turistas estacionales» cree que el turismo es beneficioso para el paisaje, mientras que única- 
mente el 25,1\% de los «residentes permanentes» piensa así $\left(\chi^{2}=19,013\right.$; $p=0,004)$. Los propietarios, tanto empadronados como no empadronados, son los más preocupados por la delincuencia (el 60,9\% y el 61,9\% respectivamente están de acuerdo con la afirmación «en los últimos años hay más delincuencia en esta zona») $\left(\chi^{2}=16,405 ; p=0,012\right)$. El $67 \%$ no regresaría a su país en caso de necesitar asistencia médica continuada, aunque debe precisarse que, si bien un 78\% del grupo de los "residentes permanentes" se quedaría en la Costa Blanca, tan sólo lo haría un 39\% de los «turistas estacionales». Los que aquí se han llamado "residentes temporales» permanecerían en un $71,4 \%$ y los "propietarios de segunda residencia", en un $46,1 \%\left(\chi^{2}=86,793\right.$; $p<0,0001)$. Así, se ponen en relación las variables objetivas de empadronamiento y tenencia de la vivienda con la percepción subjetiva sobre el sentido del lugar. De este modo, las respuestas halladas resultan coherentes teóricamente con la literatura y poseen validez estadística, lo que confirma la solidez de la tipología propuesta.

A partir de la revisión de las clasificaciones elaboradas en los últimos años y del trabajo de investigación expuesto, se ha comprobado que una tipología analítica basada en la propiedad de la vivienda y en el hecho de estar o no empadronado se muestra válida para ayudar a esclarecer el significado del complicado proceso turístico-residencial. En el cuadro 2, se sistematizan las características de las cuatro categorías identificadas.

\section{Conclusiones}

En la ciudadanía de las sociedades europeas más avanzadas, ha brotado un sentimiento de nostalgia por paisajes y modos de vida difíciles de hallar en sus entornos habituales, lo que ha contribuido al surgimiento de nuevas formas de turismo y al incremento de las migraciones por motivos residenciales. Es el caso de los europeos en situación de inactividad laboral que se trasladan de forma más o menos definitiva a la provincia de Alicante en busca de un imaginado «estilo de vida mediterráneo» (Huete, 2005, 2009).

Distintos autores han prestado una atención específica a la formulación de tipologías para definir el proceso de las migraciones internacionales de retirados y el llamado turismo residencial. Algunos investigadores han utilizado las mismas variables (empadronamiento y tenencia del alojamiento) que se han empleado para la elaboración de la tipología presentada, pero no realizaron pruebas estadísticas para su contraste empírico. La posición aquí defendida en el debate sobre las relaciones entre el turismo y la migración se concreta en la delimitación de las expresiones «inmigrante» $\mathrm{y}$ «turista». El término inmigrante, al implicar un cambio de la residencia y del contexto en el que tiene lugar el conjunto de actividades que lleva a cabo una persona en su vida cotidiana (LiviBacci, 1981), podría ajustarse a los tipos de ciudadanos que están empadronados, sean o no propietarios de su vivienda, pero no a los «propietarios de segunda residencia» (no empadronados), quienes, en muchos casos, pasan en España temporadas relativamente cortas y mantienen una vivienda principal 
en sus países de origen. Menos aún, se puede aplicar a quienes, alojándose en una vivienda privada, ni son los propietarios de la misma ni están registrados, a pesar de permanecer un cierto tiempo en la región.

En este artículo, se ha hecho referencia al concepto «turismo residencial» ante la falta de consenso entre los académicos para caracterizar este complejo fenómeno social. Se ha decidido echar mano de esta imprecisa expresión porque, en los últimos años, se ha consolidado entre la ciudadanía, como prueban recientes investigaciones orientadas a analizar la percepción del cambio social vinculado al hecho turístico en las regiones mediterráneas (Huete et al., 2008; Mantecón, 2008a, 2008b). Esta expresión, si bien recoge la idea de que los residentes noreuropeos en el Mediterráneo convierten de alguna manera el turismo en su modus vivendi (O'Reilly, 2007a), describe únicamente a una parte de los ciudadanos objeto de este estudio, ya que no se puede llamar «turistas» a quienes se instalan definitivamente en un lugar. Quizás la perspectiva teórica de la movilidad (Urry, 2007) pueda consolidarse como un argumento alternativo capaz de abordar tanto los movimientos turísticos como las migraciones, de modo que se superen las limitaciones mostradas por los paradigmas explicativos tradicionales para analizar procesos como el aquí tratado. En este sentido, se propone reservar la expresión "turistas» para aquellas personas que quieren volver, es decir, «están de paso» disfrutando de sus vacaciones en una vivienda secundaria de su propiedad o en cualquier otro régimen, y denominar «inmigrantes» o, en todo caso, «residentes» a los ciudadanos que no quieren o no saben si volverán a sus países de origen en un futuro predecible, y que lo demuestran empadronándose en los correspondientes registros.

Por otro lado, un interesante planteamiento teórico encaminado a lograr la distinción acerca de cuándo una vivienda es turística es el basado en el significado subjetivo que cada persona otorga al uso que da a la vivienda (Callejo et al., 2004), es decir, que sea el morador de la vivienda quien se califique como turista o inmigrante (Aronsson, 2004; Duval, 2004) según sus lazos de pertenencia y apego (Gustafson, 2002a). Ello, no obstante, hace necesario el establecimiento de unos indicadores que permitan la medición. Un ciudadano que se empadrona en una población determinada y que compra una vivienda allí desarrolla unos lazos de pertenencia al lugar más fuertes que quien vive de alquiler y sin estar sometido a las regulaciones burocráticas. El mantenimiento de una casa en el país de origen, la propiedad de la vivienda y el empadronamiento han demostrado ser variables operativas con las que poder manejarse en próximos trabajos, frente a otras de difícil medición, como el apego al lugar y el motivo para el traslado.

La población objeto de estudio es protagonista de un traslado más o menos temporal asociado a motivaciones que tienen que ver más con una función vinculada al ocio que con la actividad productiva. Se podría sostener que quienes compran una vivienda con el fin de utilizarla como segunda residencia, o para el traslado tras la jubilación, se sienten atraídos por el destino por las mismas razones que quienes vienen a pasar unos días de vacaciones: un clima beneficioso para la salud, una buena combinación del entorno natural y social, y 
un precio asequible. Por lo tanto, sería más adecuado adoptar la expresión "movilidad residencial por motivos de ocio" para referirnos al proceso social del que forman parte los cuatro tipos identificados.

Esos cuatro tipos han sido analizados y presentados aquí como una clasificación operativa para investigar el complicado entramado residencial que caracteriza a muchas de las poblaciones turísticas, o de aquellas otras que se hallan en su proximidad, localizadas en las costas mediterráneas. Un entramado que, hasta ahora, se ha denominado «turismo residencial», tanto en diversos ámbitos científicos, como por la ciudadanía, influida por los intereses comerciales de los promotores inmobiliarios. La clasificación puede integrarse como una herramienta de utilidad en los sistemas de gestión de recursos y planificación regional de extensos territorios, así como en futuros proyectos de investigación sobre un fenómeno social con indicios de ser cada vez más importante. Más aún cuando se comprueba cómo los ayuntamientos de los municipios en los que este proceso se ha manifestado de manera más intensa se han tropezado con grandes dificultades a la hora de explicar el complejo sistema de tipos de movilidad residencial ante el que se enfrentan diariamente. La caracterización de los tipos de movilidad identificados igualmente puede ayudar a justificar los recursos necesarios para la Administración municipal de estos territorios.

\section{Referencias bibliográficas}

ARONSSON, Lars (2004). «Place attachment of vacation residents: Between tourists and permanent residents». En: HALL, C. Michael y MüLleR, Dieter K. (eds.). Tourism, mobility and second homes: Between elite landscape and common ground. Clevedon: Channel View Publications.

BARRETTO, Margarita (2008). «Interfaces entre turismo e migrações. Uma abordagem epistemológica». Pasos: Revista de Turismo y Patrimonio Cultural, 7 (1), 1-11.

Benson, Michaela y O'ReiLly, Karen (2009). «Migration and the search for a better way of life: A critical exploration of lifestyle migration». The Sociological Review, 57(4), 608-625.

BeLL, Martin y WARD, Gary (2000). «Comparing temporary mobility with permanent migration». Tourism Geographies, 2 (1), 87-107.

BETTY, Charles y CAHILL, Michael (1998). «Consideraciones sociales y sanitarias sobre los inmigrantes británicos mayores en España, en particular los de la Costa del Sol». Migraciones, 3, 83-115.

BREuER, Toni (2005). «Características del turismo residencial de alemanes jubilados: resultados de una encuesta realizada en las Islas Canarias». En: MAZÓN, Tomás y ALEDO, Antonio (eds.). Turismo residencial y cambio social: Nuevas perspectivas teóricas y empiricas. Alicante: Aguaclara.

Callejo, Javier; GutiérReZ, Jesús y Viedma, Antonio (2004). Transformaciones de la demanda turística española: apuntes prácticos. Madrid: Ramón Areces.

CASADO, Ma Ángeles (1999). «Socio-demographic impacts of residential tourism: A case study of Torrevieja, Spain». International Journal of Tourism Research, 1, 223-237.

Casado, Ma Ángeles; Kaiser, Claudia y Warnes, Anthony (2004). «Northern European retired residents in nine southern European areas: characteristics, motivations and adjustment». Ageing and Society, 24 (3), 353-381. 
DeHOORNE, Olivier (2002). «Tourisme, travail, migration: interrelations et logiques mobilitaires». Revue Européenne des Migrations Internationales, 18 (1), 7-36.

Duval, David Timothy (2004). "Mobile migrants: Travel to second homes». En: Hall, C. Michael y Müller, Dieter K. (eds.). Tourism, mobility and second homes: Between elite landscape and common ground. Clevedon: Channel View Publications.

Gustafson, Per (2002a). "Tourism and seasonal retirement migration». Annals of Tourism Research, 29 (4), 899-918.

- (2002b). Place attachment and mobility: Three sociological studies. Göteborg: Göteborg University. Department of Sociology.

Hall, C. Michael y Müller, Dieter K. (eds.) (2004). Tourism, mobility and second homes: Between elite landscape and common ground. Clevedon: Channel View Publications.

HuBER, Andreas (2005). «La migración de retirados entre culturas: El caso de los jubilados suizos en la Costa Blanca». En: MAzón, Tomás y Aledo, Antonio (eds.). Turismo residencial y cambio social: Nuevas perspectivas teóricas y empiricas. Alicante: Aguaclara.

HuETE, Raquel (2005). «Looking for paradise: images of Spanish lifestyle». En: BuRNS, Peter (dir.). The end of tourism? Mobility and local global connections. Eastbourne: University of Brighton.

- (2009). Turistas que llegan para quedarse: Una explicación sociológica sobre la movilidad residencial. Alicante: Publicaciones de la Universidad de Alicante.

Huete, Raquel; Mantecón, Alejandro y Mazón, Tomás (2008). «¿De qué hablamos cuando hablamos de turismo residencial?». Cuadernos de Turismo, 22, 101-121.

JAAKSON, Reiner (1986). «Second-home domestic tourism». Annals of Tourism Research, 13, 357-391.

JURDAO, Francisco (1979). España en venta: compra de suelo por extranjeros y colonización de campesinos en la Costa del Sol. Madrid: Ayuso.

KING, Russell (2002). "Towards a new map of European migration». International Journal of Population Geography, 8, 89-106.

KING, Russell; WARNES, Anthony y WiLLIAMS, Allan (1998). «International Retirement Migration in Europe». International Journal of Population Geography, 4, 91-111.

- (2000). Sunset lives: British retirement migration to the Mediterranean. Oxford: Berg.

La PARra, Daniel y MATEO, Miguel Ángel (2008). «Health status and access to health care of British nationals living on the Costa Blanca, Spain». Ageing and society, 28 (1), 85-102.

LEONTIDOU, Lila y MARMARAS, Emmanuel (2001). «From tourists to migrants. Residential tourism and "littoralization"». En: Apostolopoulos, Yiorgos; LOUKISSAS, Philippos y LEONTIDOU, Lila (eds.). Mediterranean tourism. Facets of socioeconomic development and cultural change. Londres: Routledge.

LiVI-BACCI, Massimo (1981). Introducción a la demografía. Barcelona: Ariel (1 ${ }^{\mathrm{a}} \mathrm{ed}$. en castellano de 1993).

MANTECÓN, Alejandro (2008a). La experiencia del turismo: Un estudio sociológico sobre el proceso turístico-residencial. Barcelona: Icaria.

- (2008b). «Procesos de urbanización turística: Aproximación cualitativa al contexto ideológico". Papers. Revista de Sociología, 89, 127-144.

MAZÓN, Tomás (2006a). "Inquiring into residential tourism: the Costa Blanca case». Tourism and Hospitality Planning \& Development, 3 (2), 89-97. 
- (2006b). «El turismo litoral mediterráneo: ¿políticas turísticas o desarrollo inmobiliario?». En: RodRíGUEZ, Josep A. (ed.). Sociología para el futuro. Barcelona: Icaria.

MAZÓn, Tomás y Aledo, Antonio (eds.) (2005). Turismo residencial y cambio social: Nuevas perspectivas teóricas y empiricas. Alicante: Aguaclara.

MONREAL, Juan (dir.) (2001). Un nuevo mercado turístico: jubilados europeos en la región de Murcia. Murcia: Universidad de Murcia.

Moreno, Raquel; AlCALde, Rosalina y Lurbe, Kàtia (2004). «Emigrar en la madurez, inmigrar en la vejez: Las condiciones de vida de los inmigrados jubilados extranjeros en las costas mallorquinas y catalanas». Actas del IV Congreso sobre la inmigración en España. Universitat de Girona. CD-ROM.

MÜLLER, Dieter K. (2002). «German second homeowners in Sweden: Some remarks on the tourism-migration-nexus». Revue Européenne des Migrations Internationales, 18 (1), 67-86.

O'ReILly, Karen (1995). "A new trend in European migration: contemporary British migration to Fuengirola, Costa del Sol». Geographical Viewpoint, 23, 25-36.

- (2000). The British on the Costa del Sol: Transnational identities and local communities. Londres: Routledge.

- (2003). "When is a tourist?: The articulation of tourism and migration in Spain's Costa del Sol». Tourist Studies, 3 (3), 301-317.

- (2005). «Los jubilados británicos en la Costa del Sol». En: RodrígueZ, Vicente; Casado, Ma Ángeles y Huber, Andreas (eds.). La migración de europeos retirados en España. Madrid: CSIC.

- (2007a). «Emerging tourism futures: Residential tourism and its implications». En: GeOfFrey, Christine y Sibley, Richard (eds.). Going abroad: Travel, tourism, and migration. Newcastle: Cambridge Scholars Publishing.

- (2007b). «Intra-European migration and the mobility-enclosure dialectic». Sociology, 41 (2), 277-293.

RayA, Pedro y Benítez, José J. (2002). «Concepto y estimación del turismo residencial: aplicación en Andalucía». Papers de Turisme, 31/32, 67-89.

RODRÍGUEZ, Vicente (2001). «Tourism as a recruiting post for retirement migration». Tourism Geographies, 3 (1), 52-63.

- (2004). "Turismo residencial y migración de jubilados». En: Aurioles, Joaquín (coord.). Las nuevas formas de turismo. Almería: Instituto de Economía y Geografía. Caja Rural Intermediterránea. Cajamar.

RodrígueZ, Vicente; Fernández-Mayoralas, Gloria y Rojo, Fermina (2004). "International retirement migration: Retired Europeans living on the Costa del Sol, Spain». Population Review, 43 (1), 1-36.

RodrígueZ, Vicente; CASAdO, Ma Ángeles y Huber, Andreas (eds.) (2005). La migración de europeos retirados en España. Madrid: CSIC.

SALVÀ, Pere (2002). "Foreign immigration and tourism development in Spain's Balearic Islands». En: Hall, C. Michael y Williams, Allan (eds.). Tourism and migration: New relationships between production and consumption. Londres: Kluwer Academic Publishers.

SERrANO, José María (2007). «El turismo residencial en la Región de Murcia frente a los nuevos retos». Cuadernos de Turismo, 19, 189-216.

Thumerelle, Pierre-Jean (1986). Peuples en mouvement: La mobilité spatiale des populations. París: Sedes.

TORRES BERNIER, Enrique (2003). «El turismo residenciado y sus efectos en los destinos turísticos». Estudios Turísticos, 155/156, 45-70. 
URRY, John (2007). Mobilities. Cambridge: Polity.

VERA, Fernando (1990). «Turismo y territorio en el litoral mediterráneo español». Estudios Territoriales, 32, 81-110.

- (2005). «El auge de la función residencial en destinos turísticos del litoral mediterráneo: entre el crecimiento y la renovación». Papers de Turisme, 37/38, 95-114.

Warnes, Anthony; Friedrich, Klaus; Kellaher, Leonie y Torres, Sandra (2004). "The diversity and welfare of older migrants in Europe». Ageing and Society, 24, 307-326.

Williams, Allan y Hall, C. Michael (2000). «Tourism and migration: New relationships between production and consumption». Tourism Geographies, 2 (1), 5-27.

- (2002). "Tourism, migration, circulation and mobility: The contingencies of time and place». En: HALL, C. Michael y Williams, Allan (eds.). Tourism and migration. New relationships between production and consumption. Londres: Kluwer Academic Publishers.

Williams, Allan; King, Russell y Warnes, Anthony (1997). «A place in the sun: International retirement migration from northern to southern Europe». European Urban and Regional Studies, 4, 115-134.

Williams, Allan; King, Russell; Warnes, Anthony y Patterson, Guy (2000). «Tourism and international retirement migration: New forms of an old relationship in southern Europe». Tourism Geographies, 2 (1), 28-49. 